diagnosis of genetically heterogeneous disorders such as the hereditary ataxias.

\section{IN PRAISE OF ANELOVIRUSES, SAVIOURS OF THE NUCLEOTIDE ECONOMY: A CASE BASED ON EVIDENCE FROM SYNTHETIC BIOLOGY AND GENETICS}

Tim Shaw ${ }^{1}$, Andrew Peel ${ }^{2}$

${ }^{1}$ Victorian Infectious Diseases Reference Laboratory, The Doherty Institute, and ${ }^{2}$ Department of Computing and Information Systems, The University of Melbourne, Melbourne, Vic, Australia

Synthetic biology aims to mimic complex biological networks using electronic equivalents and vice versa, an idea inspired by the recognition of striking similarities between evolved (genetic) and engineered (computational) control systems. In this context, viral infection is analogous to re-programming a control system by adding a regulatory module ('modulon') to change the performance of the entire network.

The recently recognised anellovirus family comprises four genera of small single-stranded DNA viruses typified by genetic heterogeneity, broad tissue tropism and exceptionally high global distribution. Human torque teno virus (TTV), the founding member, was discovered in 1997 and subsequently associated with a wide variety of pathological conditions but without convincing evidence for a causal role in disease aetiology. Widespread chronic anelloviruses infection has now been detected in all families of ectothermic vertebrates (mammals and birds) that have been tested. Infection is generally asymptomatic, suggesting that it might confer a survival advantage.

A simple electronic circuit model was designed to mimic anellovirus infection. Input data was obtained from recent publications. The model predicts that during physiological stress, TTV replication provides a temporary nucleotide bank, saving energy by rescuing nucleotides from catabolism. TTV could therefore be regarded as a viral commensal.

\section{THE IMPORTANCE OF REPEATING A BONE MARROW EXAMINATION - A FATAL CASE OF EPSTEIN-BARR VIRUS (EBV) DRIVEN HAEMOPHAGOCYTIC LYMPHOHISTIOCYTOSIS (HLH) IN A 1 YEAR OLD}

Rebecca Barton, Anthea Greenway, Janine Campbell Royal Children's Hospital, Department of Haematology, Melbourne, Vic, Australia

Aim: To describe progressive bone marrow changes in a case of EBV associated HLH in a 1 year old.

Methods: Retrospective case review

Results: This case review describes a previously well, immunised 1-year-old girl, who presented with a 5 day history of fever with no focus. On examination she was febrile, tachycardic with hepatomegaly. Initial investigations demonstrated severe pancytopenia, coagulopathy, positive EBV serology and deranged liver function tests. The patient was commenced on high dose broad spectrum antibiotics with additional investigations revealing a metabolic acidosis and elevated ferritin. A bone marrow aspirate (BMA) showed a myeloid left shift with minimal haemophagocytosis. Despite inconclusive bone marrow findings a probable diagnosis of EBV driven HLH was made and Alemtuzumab was commenced. A repeat BMA 24 hours later showed rapid progression with florid haemophagocytosis. Further results demonstrated an elevated soluble CD25. Clinical course was complicated by rapid disease progression with multiorgan failure. Despite maximal treatment there was no improvement in disease burden and care was withdrawn.

Conclusion: Infection associated HLH can have a rapidly progressive course. Morphological changes in the BMA may lag behind clinical deterioration and confirmatory results such as CD25 may not be immediately available. Repeat BMA if clinical suspicion is high has an important role in confirming the diagnosis. References

1. Gupta A, Tyrell P, Valani R, et al. The role of the initial bone marrow aspirate in the diagnosis of haemophagocytic lymphohistiocytosis. $P e$ diatric Blood Cancer 2008; 51: 402-4.

2. Jordan MB, Allen CE, Weitzman S, et al. How I treat haemophagocytic lymphohistiocytosis. Blood 2011; 118: 4041-52.

\section{ORAL ANTICOAGULANT THERAPY INTERRUPTION IN CHILDREN: A SINGLE CENTRE EXPERIENCE}

\section{Sally Campbell, Paul Monagle, Fiona Newell}

Clinical Haematology - Paediatric Thrombosis and

Haemostasis, Royal Children's Hospital, Melbourne, Vic, Australia

\begin{abstract}
Aim and background: The use of anticoagulant therapy in paediatrics is common and there is a weak evidence base behind many of the recommendations for anticoagulant therapy in paediatric patients. To date institutional protocols for bridging anticoagulation are based on adult guidelines. This study seeks to review the clinical outcomes associated with current bridging practices employed at a tertiary metropolitan children's hospital. Methods: The patient population was selected from the warfarin management registry of a Clinical Haematology service. Data relating to demographics, anticoagulant therapy interruption plan, and clinical outcomes were recorded.

Results: A total of 61 admissions for children aged between 1 year and 17 years and 11 months were analysed for this study. Congenital heart disease (CHD) was the primary underlying disease for which long-term oral anticoagulation with warfarin was indicated. There were no instances of major bleeding $(n=0)$ or thrombotic events $(n=0)$.

Conclusion: This study describes the current practices and outcomes associated with anticoagulant therapy interruption at one paediatric institution representing the largest and most representative cohort to date. This project is a stepping stone from which future studies of safety and efficacy of paediatric anticoagulation interruption management can be developed.
\end{abstract}

\section{EVALUATION OF SNP MICROARRAY BASED CHROMOSOME TESTING FOR DETECTION OF PROGNOSTICALLY RELEVANT GENOMIC ABNORMALITIES IN CHRONIC LYMPHOCYTIC LEUKAEMIA}

Melody Caramins ${ }^{1}$, Nicole Chia ${ }^{2}$, Stephen Mulligan

${ }^{1}$ Specialist Diagnostic Services (SDS Pathology), University of New South Wales, NSW, ${ }^{2}$ QML Pathology, Qld, and ${ }^{3}$ University of Sydney, NSW, Australia 